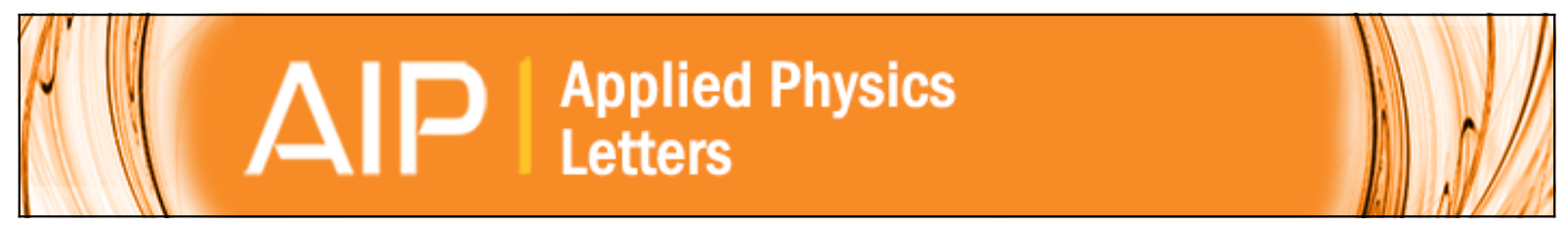

\title{
Effect of grain alignment on interface trap density of thermally oxidized aligned- crystalline silicon films
}

Woong Choi, Jung-Kun Lee, and Alp T. Findikoglu

Citation: Applied Physics Letters 89, 262111 (2006); doi: 10.1063/1.2424655

View online: http://dx.doi.org/10.1063/1.2424655

View Table of Contents: http://scitation.aip.org/content/aip/journal/apl/89/26?ver=pdfcov

Published by the AIP Publishing

\section{Articles you may be interested in}

Fixed nitrogen atoms in the $\mathrm{SiO} 2 / \mathrm{SiC}$ interface region and their direct relationship to interface trap density Appl. Phys. Lett. 99, 182111 (2011); 10.1063/1.3659689

A model of electrical conduction across the grain boundaries in polycrystalline-silicon thin film transistors and metal oxide semiconductor field effect transistors

J. Appl. Phys. 106, 024504 (2009); 10.1063/1.3173179

Effects of anneals in ammonia on the interface trap density near the band edges in $4 \mathrm{H}-$ silicon carbide metaloxide-semiconductor capacitors

Appl. Phys. Lett. 77, 3601 (2000); 10.1063/1.1328370

Effect of nitric oxide annealing on the interface trap densities near the band edges in the $4 \mathrm{H}$ polytype of silicon carbide

Appl. Phys. Lett. 76, 1713 (2000); 10.1063/1.126167

Measurement of interface trap states in metal-ferroelectric-silicon heterostructures

Appl. Phys. Lett. 72, 2283 (1998); 10.1063/1.121337

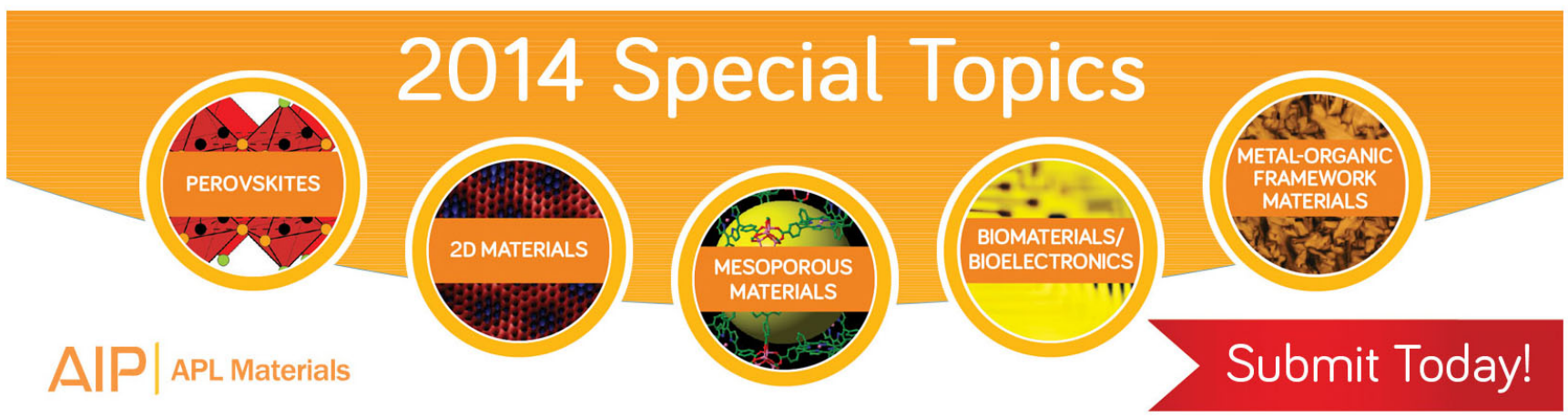




\title{
Effect of grain alignment on interface trap density of thermally oxidized aligned-crystalline silicon films
}

\author{
Woong Choi, ${ }^{\text {a) }}$ Jung-Kun Lee, and Alp T. Findikoglu ${ }^{\text {b) }}$ \\ Materials Physics and Applications Division, Los Alamos National Laboratory, \\ Los Alamos, New Mexico 87545
}

(Received 7 August 2006; accepted 28 November 2006; published online 28 December 2006)

\begin{abstract}
The authors report studies of the effect of grain alignment on interface trap density of thermally oxidized aligned-crystalline silicon (ACSi) films by means of capacitance-voltage $(C-V)$ measurements. $C$ - $V$ curves were measured on metal-oxide-semiconductor (MOS) capacitors fabricated on $\langle 001\rangle$-oriented ACSi films on polycrystalline substrates. From high-frequency $C$ - $V$ curves, the authors calculated a decrease of interface trap density from $2 \times 10^{12}$ to 1 $\times 10^{11} \mathrm{~cm}^{-2} \mathrm{eV}^{-1}$ as the grain mosaic spread in ACSi films improved from $13.7^{\circ}$ to $6.5^{\circ}$. These results demonstrate the effectiveness of grain alignment as a process technique to achieve significantly enhanced performance in small-grained $(\leqslant 1 \mu \mathrm{m})$ polycrystalline Si MOS-type devices. (C) 2006 American Institute of Physics. [DOI: 10.1063/1.2424655]
\end{abstract}

Interface trap density is a critical parameter that affects the electronic properties of oxidized semiconductor surfaces, and thus the performance of various electronic devices, such as metal-oxide-semiconductor (MOS) field effect transistors. ${ }^{1}$ For single-crystal Si-based MOS devices, the interface trap density has been known to depend on the oxidation process, substrate orientation, and interface roughness. ${ }^{2,3}$ While MOS devices based on polycrystalline Si thin films have been of great interest for applications in thin-film transistors (TFTs), little attention has been given to the relationship between interface trap density and grain alignment in polycrystalline Si films. Recently, we reported a technique that uses biaxially oriented buffer layers to grow $\langle 011\rangle$ oriented Si thin films, called aligned-crystalline Si (ACSi), on flexible polycrystalline metal tapes with controllable grain alignment. $^{5-7}$ For the work presented here, we have fabricated MOS capacitors from $\langle 001\rangle$-oriented ACSi films on flexible substrates and investigated the correlation between interface trap densities and grain alignment by using capacitance-voltage $(C-V)$ measurements.

The ACSi films were grown on polycrystalline metal tapes (Hastelloy C-276, $100 \mu \mathrm{m}$ thick) using a buffer layer architecture composed of $\gamma-\mathrm{Al}_{2} \mathrm{O}_{3}, \mathrm{MgO}$, and $\mathrm{Y}_{2} \mathrm{O}_{3}$. Details on growth conditions can be found elsewhere. ${ }^{5}$ All the layers were deposited by electron-beam evaporation in the following sequence: a $\mathrm{Y}_{2} \mathrm{O}_{3}$ nucleation layer $(5 \mathrm{~nm})$ at room temperature, a biaxially oriented $\mathrm{MgO}$ layer $(10 \mathrm{~nm})$ using an ion-assist beam of $750 \mathrm{eV} \mathrm{Ar}^{+}$at room temperature, a homoepitaxial $\mathrm{MgO}$ layer $(100-200 \mathrm{~nm})$ at $500{ }^{\circ} \mathrm{C}$, and a $\gamma-\mathrm{Al}_{2} \mathrm{O}_{3}$ buffer layer $(100-200 \mathrm{~nm})$ at $800{ }^{\circ} \mathrm{C}$. A heavily doped $p^{+}$-ACSi bottom electrode layer $(1 \mu \mathrm{m})$, followed by a lightly doped $p$-ACSi layer $(3 \mu \mathrm{m})$, was grown at $710-770{ }^{\circ} \mathrm{C}$ using two separate boron-doped Si sources. The doping concentrations of $p^{+}$- and $p$-ACSi layers were measured to be $2 \times 10^{19}$ and $3 \times 10^{17} \mathrm{~cm}^{-3}$, respectively, by secondary ion mass spectroscopy (SIMS).

\footnotetext{
${ }^{\text {a) }}$ Present address: Applied Materials Inc., Sunnyvale, CA 94085; electronic mail: woong_choi@amat.com

${ }^{b)}$ Electronic mail: findik@lanl.gov
}

The crystallographic properties of the ACSi layers were analyzed by x-ray diffraction (XRD). A $\theta-2 \theta$ XRD scan of the multilayer indicated that the $\mathrm{MgO}, \gamma-\mathrm{Al}_{2} \mathrm{O}_{3}$, and $\mathrm{ACSi}$ layers grew with $\langle 001\rangle$ orientation perpendicular to the substrate surface. For the $\mathrm{Si}(004)$ reflection, the full width at half maximum (FWHM) of the rocking curve $\Delta \omega_{004}$ was in the range of $2.0^{\circ}-4.0^{\circ}$ for the series. The fourfold in-plane symmetry of the Si layer was confirmed by XRD $\phi$ scan of the 022 reflection. The average FWHM $\Delta \phi_{022}$ was distributed between $6.1^{\circ}$ and $13.1^{\circ}$. The average grain mosaic spread of each sample was analyzed using the total mosaic spread (TMS) defined as TMS $=\left(\Delta \omega_{x}^{2}+\Delta \omega_{y}^{2}+\Delta \phi_{z}^{2}\right)^{1 / 2}$, where $\Delta \omega_{x}, \Delta \omega_{y}$, and $\Delta \phi_{z}$ are the tilt along $x$, tilt along $y$, and rotation along $z$, respectively. ${ }^{5}$ The crystallographic properties were also confirmed by electron backscattering diffraction. XRD analysis indicated compressive stress in ACSi films, which was also observed by Raman spectroscopy. The root mean squared (rms) surface roughness of ACSi films was measured by atomic force microscopy on a $5 \times 5 \mu \mathrm{m}^{2}$ scale.

To form MOS capacitors, silicon oxide layers $(\sim 25 \mathrm{~nm})$ were thermally grown at $900{ }^{\circ} \mathrm{C}$ for $1 \mathrm{~h}$ in a conventional tube furnace using dry $\mathrm{O}_{2}$. Before oxidation, samples were cleaned with Radio Corporation of America (RCA) cleaning procedure ${ }^{8}$ and the native oxide layer was removed with a buffered HF solution. The FWHM of the Raman bandwidth of the thermally oxidized ACSi films was measured to analyze the defect density. MOS capacitors were patterned using standard photolithography and reactive ion etch. The sputtered Al contact electrodes $\left(80 \times 80 \mu \mathrm{m}^{2}\right.$, $200 \mathrm{~nm}$ thick) were patterned by a lift-off technique, followed by annealing in forming gas $\left(94 \% \mathrm{Ar}, 6 \% \mathrm{H}_{2}\right)$ at $450{ }^{\circ} \mathrm{C}$ for $30 \mathrm{~min}$. The $C$ - $V$ characteristics were measured at room temperature with an HP4194A impedance analyzer.

The measurement of the small signal differential capacitance of MOS capacitors as a function of gate voltage has been extensively used to extract the properties of the Si surfaces, oxide layers, and interfaces. Figure 1 shows the MOS capacitance as a function of the applied voltage measured at three different frequencies $(1,10$, and $100 \mathrm{kHz})$. It also shows a schematic cross section of the MOS capacitor. The 


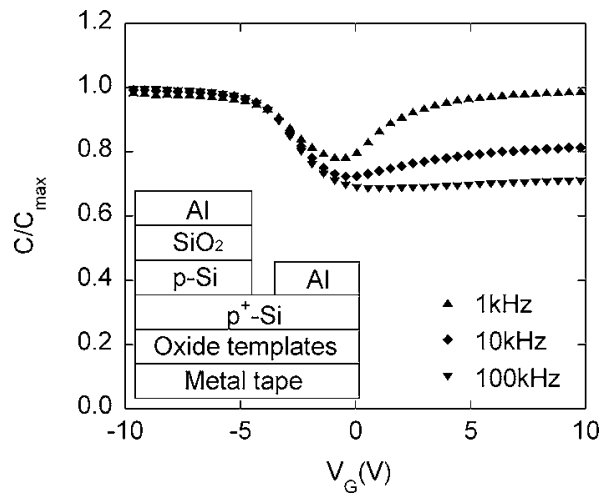

FIG. 1. Normalized capacitance $\left(C / C_{\max }\right)$ of metal-oxide-semiconductor capacitors as a function of applied gate voltage measured at 1, 10, and $100 \mathrm{kHz}$. Also shown is a schematic cross section of the MOS capacitor structure.

$C-V$ results show well defined regions of accumulation, depletion, and inversion. An accumulation capacitance appears at negative voltages, indicating that the top layer of the ACSi film is $p$ type. Figure 1 also shows typical high- and low-frequency curves at 100 and $1 \mathrm{kHz}$, respectively, with a transition curve at $10 \mathrm{kHz}$. A hole concentration $(\sim 3$ $\times 10^{17} \mathrm{~cm}^{-3}$ ) estimated from the $C-V$ curve was consistent with the doping concentration measured by SIMS. The thickness of depletion layer $(\sim 40 \mathrm{~nm})$ was also estimated from the $C$ - $V$ curve and was in good agreement with a known value at $\sim 3 \times 10^{17}$ holes $\mathrm{cm}^{-3}$ (Ref. 9).

Figure 2 shows high-frequency ( $1 \mathrm{MHz}) C-V$ curves of MOS capacitors based on ACSi films with TMS values of $6.5^{\circ}, 8.2^{\circ}, 11.9^{\circ}$, and $13.7^{\circ}$. As the TMS value increases, the $\mathrm{C}-\mathrm{V}$ curve stretches out further along the voltage axis, indicating higher density of interface traps. ${ }^{9}$ Interface traps change their charge state depending on whether they are filled or empty. Because interface trap occupancy varies with gate bias, the stretch-out of $C$ - $V$ curves occurs. To determine the correlation between interface traps and TMS in our ACSi films, we calculated the midgap interface trap density $\left(D_{\mathrm{it}}\right)$ of each ACSi sample by the high-frequency capacitance method. ${ }^{10}$ A uniform doping level of $3 \times 10^{17} \mathrm{~cm}^{-3}$ in all four ACSi samples, experimentally confirmed by SIMS, was used for this calculation. As shown in Fig. 3, $D_{\text {it }}$ of our Si films is reduced from $2 \times 10^{2}$ to $1 \times 10^{11} \mathrm{~cm}^{-2} \mathrm{eV}^{-1}$ as the TMS of the ACSi film decreases from $13.7^{\circ}$ to $6.5^{\circ}$. For single crystal $\mathrm{Si}$, a dry-oxygen-grown $\mathrm{SiO}_{2}$ layer has a typical $D_{\text {it }}$ in the

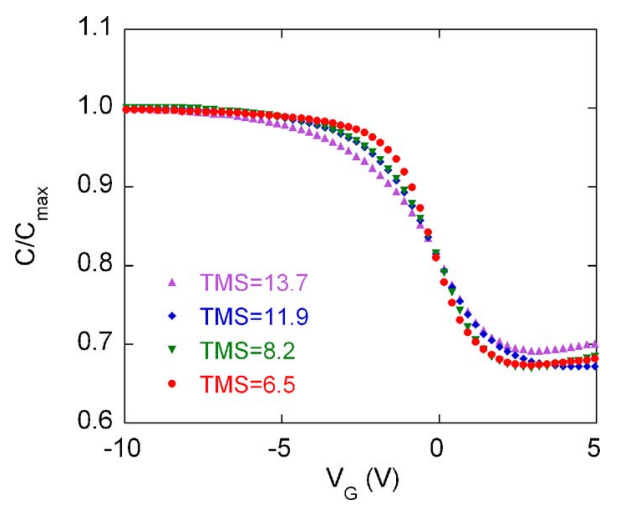

FIG. 2. (Color online) Normalized capacitance $\left(C / C_{\max }\right)$ of MOS capacitors, based on ACSi films with four different values of grain total mosaic spread (TMS), as a function of applied gate voltage measured at $1 \mathrm{MHz}$.

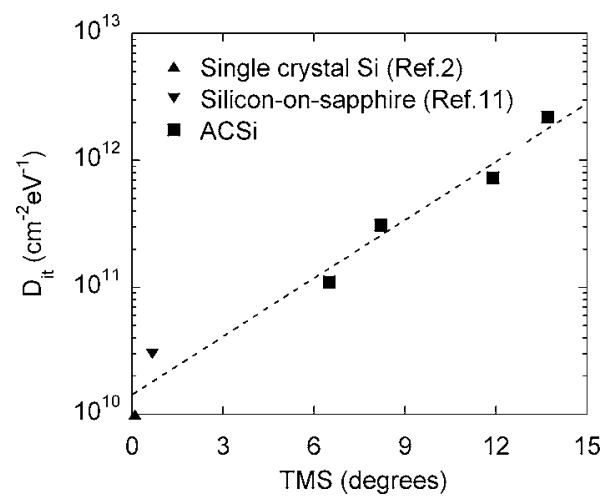

FIG. 3. Interface trap density at midgap $\left(D_{\text {it }}\right)$ as a function of total mosaic spread. $D_{\mathrm{it}}$ data of single crystal $\mathrm{Si}$ and silicon-on-sapphire are based on Refs. 2 and 11, respectively. The dashed line is a guide for the eye.

$10^{12} \mathrm{~cm}^{-2} \mathrm{eV}^{-1}$ range, but forming gas annealing reduces $D_{\text {it }}$ to the low $10^{10} \mathrm{~cm}^{-2} \mathrm{eV}^{-1}$ range. ${ }^{2}$ Consistent with the case of single crystal $\mathrm{Si}$, our thermally oxidized ACSi films also showed $D_{\mathrm{it}}$ in the $10^{12} \mathrm{~cm}^{-2} \mathrm{eV}^{-1}$ range before forming gas annealing. However, for a given series of samples, $D_{\mathrm{it}}$ could not be reduced below $1 \times 10^{11} \mathrm{~cm}^{-2} \mathrm{eV}^{-1}$ after forming gas annealing. This may be related to grain boundary traps, but it is difficult to suggest with certainty a microscopic mechanism to describe our results. Combined with $D_{\text {it }}$ data of single crystal $\mathrm{Si}$ and good-quality silicon-on-sapphire reported in the literature, ${ }^{2,11}$ these results indicate that $D_{\mathrm{it}}$ decays approximately exponentially as TMS decreases (dashed line).

Since all the samples were processed under the same conditions, changes in the oxidation process were negligible. As ACSi films showed $\langle 001\rangle$ orientation within a few degrees at the surface normal, changes of $D_{\text {it }}$ in such a narrow range of surface orientation are expected to be insignificant. ${ }^{12}$ It has also been known that $D_{\text {it }}$ increases with increasing interface roughness. ${ }^{3}$ However, while the distribution of grain size remained similar, the rms surface roughness of our ACSi films increased monotonically from 13 to $15 \mathrm{~nm}$ as the TMS decreased from $13.7^{\circ}$ to $6.5^{\circ}$, and thus cannot explain the observed $D_{\mathrm{it}}$ dependence.

Interface traps are thought to originate from dangling bonds or metallic impurities at the $\mathrm{Si}$ surfaces. ${ }^{1}$ Since we used the growth temperatures of the ACSi films as a variable to control grain alignment, we cannot exclude the possibility that changes in growth temperature might influence $D_{\text {it }}$ by significantly reducing the density of dangling bonds or defects in the Si film or at its surface. However, based on the following argument, we conclude that the effect of grain alignment on $D_{\mathrm{it}}$ is likely to be dominant over direct growth temperature effects. The experimental techniques and models employed in this study do not allow for definitive identification of the density of dangling bonds; they let us estimate the overall trap density due to all types of defects and dangling bonds. However, with forming gas anneal (FGA) studies, we have observed that the dangling bond density is likely to be negligible compared to other types of defects in wellaligned ACSi films because we have not observed any significant change in behavior with FGA in such films. Also, we measured the FWHM of the Raman bandwidth of our ACSi films to compare the defect density within Si grains (Fig. 4). The FWHM of the Raman bandwidth in polycrystalline silicon films increases with increasing defect density of the Si 


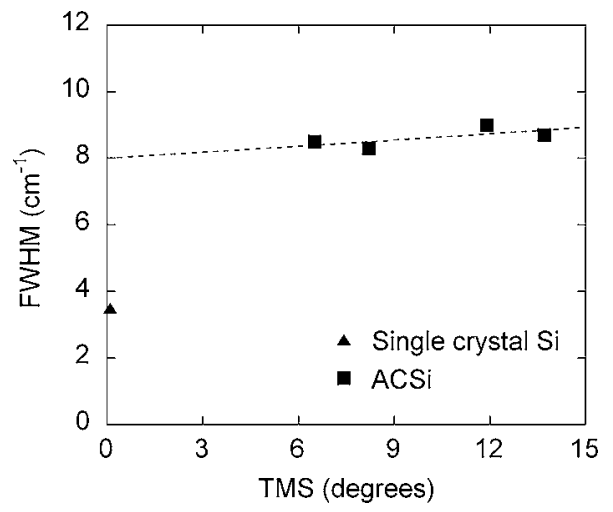

FIG. 4. Full width at half maximum (FWHM) of the Raman bandwidth of ACSi films as a function of total mosaic spread. The FWHM in single crystal $\mathrm{Si}$ was also measured as a reference. The dashed line is a guide for the eye.

grains. ${ }^{13}$ For our ACSi films, the FWHM of the Raman bandwidth varied less than $10 \%$ independent of the growth temperature, indicating that the defect density within the $\mathrm{Si}$ grains does not depend significantly on the growth temperature that we have used. For comparison, the FWHM of Raman bandwidth measured on single crystal $\mathrm{Si}$ is less than $50 \%$ of that on ACSi films.

Microstructural and electrical measurement results in previous publications already indicated that improving the grain alignment of small-grained $(\leqslant 1 \mu \mathrm{m})$ ACSi films produced effectively the same results that increasing grain size does. $^{5-7}$ This study showed that the interface trap density also decreases significantly as grain alignment improves. Therefore, ACSi process could be considered as an effective alternative to fabrication methods in TFTs, where a laser annealing method is typically used, such as sequential lateral solidification $^{14}$ or selectively enlarging laser crystallization. ${ }^{15}$ Currently, no other technology combines the highperformance aspects of single crystal silicon with the low- cost aspects of polycrystalline silicon films, which use inexpensive, non-single-crystalline substrates. As such, the ACSi process could allow the Si industry to lower its costs while maintaining or improving the performance of its products.

In conclusion, we have studied the relationship between grain alignment and interface trap density in MOS capacitors fabricated using $\langle 001\rangle$-oriented ACSi thin films on polycrystalline substrates. Based on the high-frequency capacitance method, we calculated the interface trap density, which decreased from $2 \times 10^{12}$ to $1 \times 10^{11} \mathrm{~cm}^{-2} \mathrm{eV}^{-1}$ as the grain mosaic spread decreased from $13.7^{\circ}$ to $6.5^{\circ}$. These results indicate that improved grain alignment could lead to significantly reduced interface trap density in polycrystalline Si films, with potentially important implications for TFT applications.

${ }^{1}$ S. Wolf, Silicon Processing for VLSI Era: the Submicron MOSFET (Lattice, Sunset Beach, 1995), Vol. 3, pp. 122-127.

${ }^{2}$ E. H. Nicollian and J. R. Brews, MOS (Metal Oxide Semiconductor) Physics and Technology (Wiley, New York, 1982), pp. 775-829.

${ }^{3}$ L. Lai, K. J. Hebert, and E. A. Irene, J. Vac. Sci. Technol. B 17, 53 (1999).

${ }^{4}$ S. D. Brotherton, Semicond. Sci. Technol. 10, 721 (1995).

${ }^{5}$ A. T. Findikoglu, W. Choi, V. Matias, T. G. Holesinger, Q. X. Jia, and D. E. Peterson, Adv. Mater. (Weinheim, Ger.) 17, 1527 (2005).

${ }^{6}$ W. Choi, V. Matias, J. K. Lee, and A. T. Findikoglu, Appl. Phys. Lett. 87, 152104 (2005).

${ }^{7}$ W. Choi, A. T. Findikoglu, M. J. Romero, and M. Al-Jassim, J. Mater. Res. (to be published).

${ }^{8}$ W. Kern and D. A. Puotinen, RCA Rev. 31, 187 (1970).

${ }^{9}$ S. M. Sze, Physics of Semiconductor Devices (Wiley, New York, 1981), pp. 362-407.

${ }^{10}$ L. M. Termin, Solid-State Electron. 5, 285 (1962).

${ }^{11}$ I. R. Evans, IEEE Electron Device Lett. 10, 25 (1989).

${ }^{12}$ E. Arnold, J. Ladell, and G. Abowitz, Appl. Phys. Lett. 13, 413 (1968).

${ }^{13}$ T. Jawhari, Analusis 28, 15 (2000).

${ }^{14}$ J. S. Im, R. S. Sposili, and M. A. Crowder, Appl. Phys. Lett. 70, 3434 (1997).

${ }^{15}$ M. Tai, M. Hatano, S. Yamaguchi, T. Noda, S. K. Park, T. Shiba, and M. Ohkura, IEEE Trans. Electron Devices 51, 934 (2004). 